\title{
Spatial Degradation of Classified Satellite Images
}

\author{
Stella Hubert ${ }^{1}$, Stefan Schwarzer ${ }^{* 2,3}$ and Jean-Michel Jaquet ${ }^{2,3}$
}

\begin{abstract}
${ }^{I}$ Deutsches Zentrum für Luft- und Raumfahrt, Deutsches Fernerkundungsdatenzentrum, D-82234 Oberpfaffenhofen, Germany; ${ }^{2}$ United Nations Environment Programme/DEWA/GRID-Geneva, 11 chemin des Anémones, 1219 Geneva, Switzerland ; ${ }^{3}$ University of Geneva, Institute for Environmental Studies, 7 route de Drize, 1227 Carouge, Geneva, Switzerland
\end{abstract}

\begin{abstract}
One of the most common uses of classified satellite images is mapping land cover alterations via change detection. If images for two different dates are not available from the same sensor, one has to use two sensors with different resolutions, and images or maps must be either upscaled or downscaled. We present a study based on two landcover maps produced for the agglomeration of Geneva, Switzerland: one derived from orthophotos at $0.25 \mathrm{~m}$ resolution and one from a SPOT image at $5 \mathrm{~m}$. Four quantitative methods ("direct", "nearest neighbour", "majority" and "statistical") have been tested to degrade the higher-resolution map to a $5 \mathrm{~m}$ resolution with a minimum loss of information, in order to make both maps comparable. Upscaled maps have been compared with the original (a) qualitatively by visual examination, and (b) quantitatively by computing Kappa coefficients.Special attention was given to the preservation of thin linear features, such as roads. Visual inspection showed that the majority and statistical methods are unable to preserve thin linear features. With the exception of the direct method,which is the least favourable one, the others perform almost equally well according to the statistical analysis. Overall, the nearest neighbour approach yields the smallest loss of information, with Kappa values up to $92 \%$.
\end{abstract}

Keywords: Remote sensing, land cover mapping, upscaling, ArcGIS.

\section{INTRODUCTION}

Land cover is an important environmental factor, which has been used in a variety of studies such as groundwater levels [1], soil erosion [2,3], biodiversity [4] and energy budgets $[5,6]$. As ground surveys are not only timeconsuming, but also very costly, remote sensing offers an efficient alternative to deliver the necessary land cover/use information.

Land cover maps taken at different times and at similar scale are the base material for change analyses [7-9] or land cover transition estimates [11]. However, using older land cover classifications together with very recent ones will most of the time result in a combination of layers with two or more different spatial resolutions. While various authors have worked on upscaling remotely sensed data directly $[12,13]$, hardly any literature could be found which analysed and explained what approach should be used to upscale existing classifications.

This paper aims at evaluating different methods of degrading a $0.25 \mathrm{~m}$-resolution landcover map obtained by classification of orthophotos in the vicinity of Geneva, Switzerland, to a $5 \mathrm{~m}$ resolution, in order to allow comparisons with a regional land cover map derived from SPOT imagery.

\footnotetext{
*Address correspondence to this author at the United Nations Environment Programme/DEWA/GRID-Geneva, 11 chemin des Anémones, 1219 Geneva, Switzerland; Tel: 0041.22.917.83.49; Fax: 0041.22.917.80.29;

E-mail: stefan.schwarzer@unep.org
}

\section{CONTEXT AND DATA}

This study was carried out as part of the "Agglomération franco-valdo-genevoise" project [14], designed for the management of the transboundary area including Geneva city and bordering communes in the cantons Geneva and Vaud (Switzerland), as well as two departments in France (Ain, Haute-Savoie) Fig. (1). As a component of the $A G G L O$ project, a medium-resolution land cover map (5m pixels) was produced for the year 2003, based on an object-oriented classification of SPOT satellite imagery with $5 \mathrm{~m}$ (visible bands) and $10 \mathrm{~m}$ (near and shortwave infrared bands) resolution $[15,16]$. It is planned to update this map at a 5 year frequency, as a tool to monitor land cover change and the socio-economic evolution of the area (see [10], for a similar endeavour at European scale).

In parallel, and to comply with Swiss federal requirements, the Canton of Geneva has undertaken detailed land cover mapping of its own territory, yielding a highresolution map dating from 2005, based on an objectoriented classification of multi-source data (4-channel $25 \mathrm{~cm}$ resolution orthophotos, vector layers and digital elevation model; [17-19]). The map legend includes 35 land cover / land use classes, following the Swiss federal specifications. Updates are also planned at 5 year intervals. A comparison of these two maps will show how and where the land cover changed between 2003 and 2005, provided that the resolution of the $2005 \mathrm{map}(0.25 \mathrm{~m})$ is degraded to $5 \mathrm{~m}$.

To test the upscaling methodologies, three subsets were chosen on the high resolution map: the communes of Genève 
Cité $\left(3.0 \mathrm{~km}^{2}\right)$, Thônex $\left(3.8 \mathrm{~km}^{2}\right)$ and Satigny $\left(18.9 \mathrm{~km}^{2}\right)$ Fig. (1). These subsets differ in terms of their land use: Genève Cité, apart from the lake and the outflow of the Rhône River, is purely urban, whereas Satigny is dominated by agriculture. Thônex is a mixture between urban and vegetated parts, with vegetation covering almost two thirds of the total area. Fig. (2) shows the percentages of select land covers in the three regions.

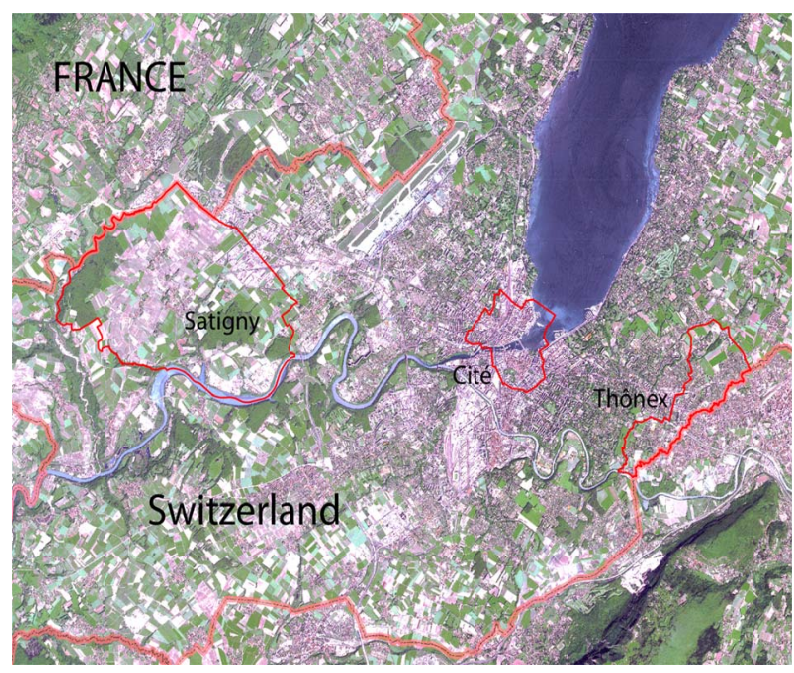

Fig. (1). Test areas outlined in red: Satigny (left), Genève Cité (middle) and Thônex (right).

During the working process, two different tests were carried out: one with the initial 35 thematic classes; another one with a reduced set of 16 classes Fig. (3). It should be noted that not all classes were present in each subset ("vineyards", for example, were not present in the Cité subset and "lake" was absent in the Thônex subset), so that on average only 26 (instead of 35) and 11 (instead of 16) classes were actually considered.

Percentages of Land Cover Type for the three Study Areas

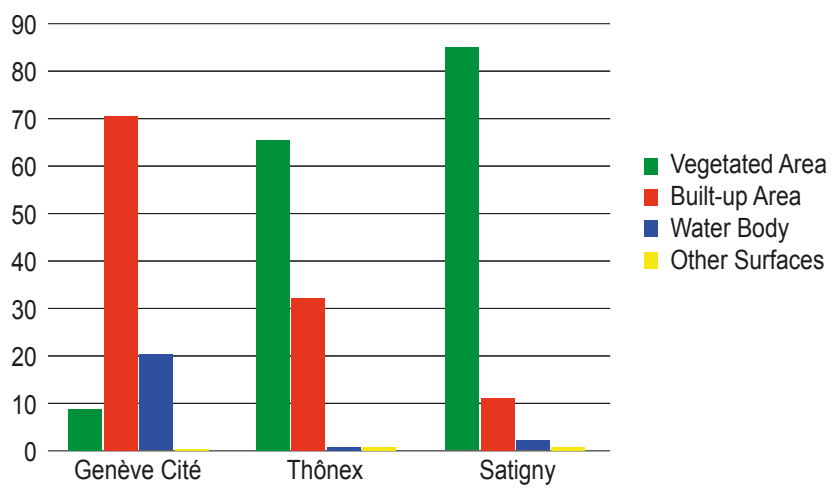

Fig. (2). Percentages of land cover type for the three study areas.

\section{METHODOLOGY}

\subsection{Upscaling}

The process of allocating new values to raster data after altering the cell size is called resampling. New values need to be assigned to the pixels in the resulting output image for two reasons:
The input grid does not necessarily align with the grid of the transformed image when the output resolution is not a multiple of the input resolution. This does not apply to our case, where the cell factor is $20(0.25 \mathrm{~m} \times 20=5 \mathrm{~m})$.

More than one pixel in the old image correspond to the new pixel in the output image. In our study, $4000.25 \mathrm{~m}$ pixels make up one $5 \mathrm{~m}$ pixel.

Fig. (4) shows an example with a cell factor of 3, nine pixels of the original image lying within one pixel of the output data set.

Four different resampling methods were tested. Three of these (the "direct method" (DM), the "nearest neighbour method" (NM) and the "majority method" (MM)) are based on predefined tools supplied by ESRI ArcMap ${ }^{\mathcal{C}}$, whereas the "statistical method" (SM) consists of a chain of different processing steps, also run under ArcMap ${ }^{\circ}$.

For all classification procedures except the "direct method", the original land cover vector dataset was first converted to a $0.25 \mathrm{~m}$ raster, keeping the original resolution of the orthophotos on which the classification was initially based.

\subsubsection{Direct Method (DM)}

The DM implies a direct conversion from the original vector land covermap to a raster of $5 \mathrm{~m}$ resolution, using the ArcToolbox Polygon to Raster tool.

\subsubsection{Nearest Neighbour Method (NM)}

In the NM method, the new value of a pixel corresponds to the value of the pixel whose centre in the input image is closest to the centre of the pixel in the output image. This operation is performed using the Arc Toolbox Resample tool, with NEAREST option. There are two possible cases: if the cell factor is odd (CF $\bmod 2 \neq 0$ ) (Fig. (5), top), the new pixel takes the unambiguous value of the center input pixel (yellow in this example). If the cell factor is even (CF mod $2=0$ ), the new pixel could take the value of any of the four original ones surrounding its centre (Fig. 5, bottom). In practice, Resample / NEAREST arbitrarily chooses the lower right one (in Orange on Fig. 5).

\subsubsection{Majority Method (MM)}

In the MM, all pixels falling into the area of the new pixel are taken into account. The most common value is then assigned to the new pixel. In case of a tie, Resample / MAJORITY seems to choose the new value at random.

As an example of an even cell factor, Fig. (6) shows a fine resolution image (upper left), which is upscaled to a coarser resolution (cell factor $=2$ ). The result of the MM is given in the upper middle box, with 3 of the upscaled cells showing ambiguous results ( grey).The other boxes depict the various possible outcomes of the NM.

\subsubsection{Statistical Method (SM)}

As mentioned above, the "statistical approach" is a chain of different processing steps. First of all, the input image is divided into blocks the size of which depends on the input and output resolutions. The majority value of each block is then calculated using Arc Toolbox Block Statistics. In Fig. (7), this is illustrated by the left images at the top and at the 


\section{Legend \\ Original Classes}

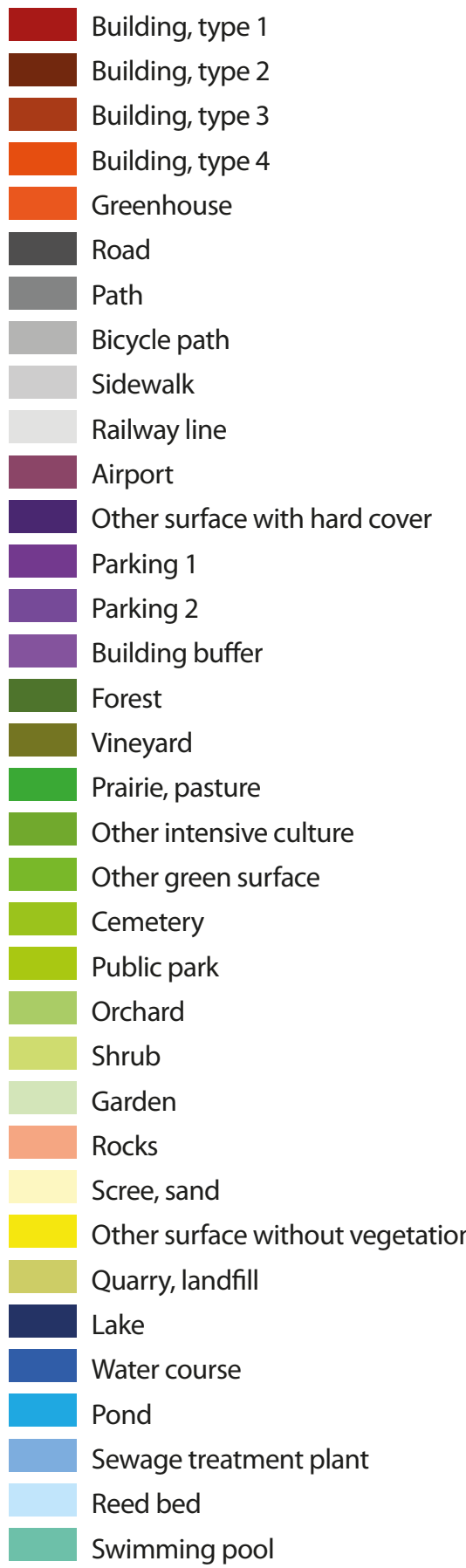

Legend

Aggregated Classes

Building

Road network

Railway line

Airport

Other surface with hard cover

\begin{tabular}{|l|}
\hline Forest \\
\hline Vineyard \\
\hline Prairie, pasture \\
\hline Other intensive culture \\
Other green surface
\end{tabular}

Rocks

Scree, sand

Other surface without vegetation

Lake

Water course

Other aquatic surfaces

Fig. (3). Thematic reduction of the classification: original classes (left) and aggregated classes.

bottom with a block size of 3 by 3 and 4 by 4, respectively. The most common value of each block, computed by the MAJORITY OPTION, is finally assigned to a new pixel (middle images). If two or more values are represented equally in one block, the value "No Data" is assigned (grey in Fig. 7). In our case, a block of 20 by 20 pixels was chosen. Note that the resolution is not altered by this operation. To do so, the data are subsequently aggregated by means of the tool Aggregate. As this process needs a function to calculate new values for each pixel, the option MEDIAN was arbitrarily chosen. If the Ignore NoData option is unchecked, there are NoData cells in the output map (shown by a question marks in Fig. (7).

One would expect the statistical and the majority methods to yield the same results. This is indeed the case when the number of columns or rows of the original image is even. If it is odd, the Resample NM and MM starts with an offset, which may slightly affect the final result. 

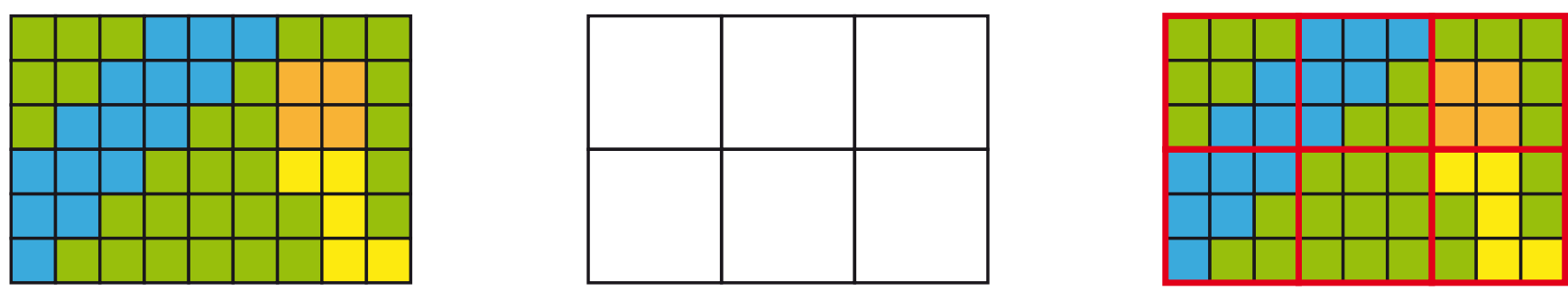

Fig. (4). Input image (left), desired new resolution (middle) and overlay of the original data with the frame of the desired resolution in red (right).

\subsection{Comparison of Different Methods}

To compare the different methods and to approximately quantify the information loss, two different kinds of accuracy assessments were carried out: one based on visual inspection, the other on a confusion matrix [20]. The analysis was undertaken with all 6 upscaled maps resulting from the combination of the 3 test areas with the 2 thematic resolutions. According to Foody [21], these two methods mark the first and the last stage of the development of accuracy assessments. As the first method mentioned is quite straight-forward, only the second one will be further explained.
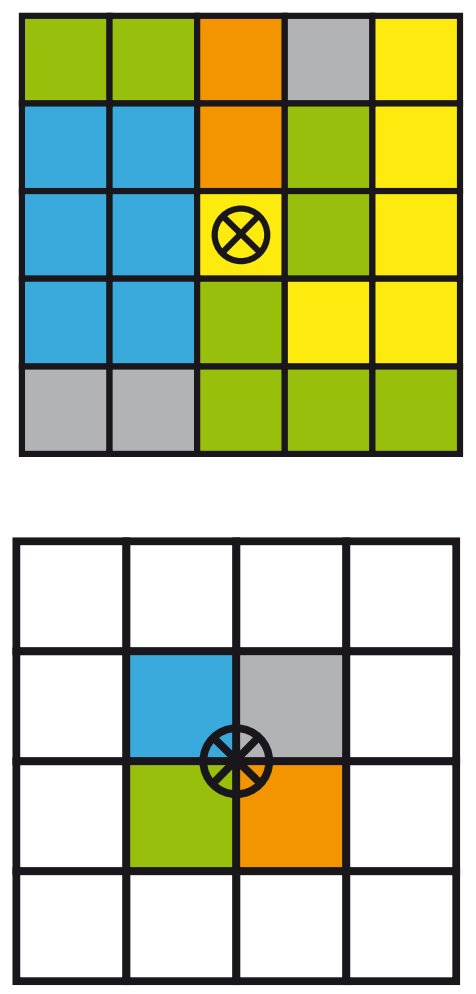

Fig. (5). Nearest-neighbour resampling in case of CF $\bmod 2 \neq 0$ (top) and $\mathrm{CF} \bmod 2=0$ (bottom). $\mathrm{CF}=$ resolution reduction factor.

Accuracy assessments are normally used to compare at least two sets of categorized data such as classifications [20]: class values are compared at the same geographic location for the different classifications. In our specific case, an error in the assessment does not indicate, as it normally does, a difference between the thematic map and reality, but between maps at different resolutions. As such, the map comparisons are better named similarity assessments, rather than accuracy assessments.

In this study, 1000 sampling points per method and thematic resolution were used. The points for each region remained the same for the classification with all initial classes and the classification with the reduced classes to allow for comparison. As the sampling points were randomly selected, the assessment was carried out four times to check for uncertainties following the chance selection of samples. Therefore, the number of sampled points actually increased to 4000. Altogether, 24 sets of statistics were computed (3 testing areas, 4 runs with different sampling points, 2 different thematic resolutions), including the following parameters: overall similarity, Kappa, the variance of Kappa and the $Z$-value of Kappa. Furthermore, for each method and each test area, the common Kappa value was computed over the different runs, as well as pair-wise $Z$ scores and $\chi^{2}$ values to test for significance.

\subsection{Problems Impacting the Quality of Accuracy Assessment}

Fig. (8) shows a 20 by 20 pixel subset of the original dataset which, after the upscaling process, is represented by only one larger pixel. This resulting pixel is illustrated according to the different methods applied. The "Other surfaces with sealed covering" (violet) and "Forest" (dark green) classes both account for 161 pixels in the original dataset, while "Other green surfaces" (light green) and "Roads" (grey) make up 41 and 37 pixels, respectively. As one can see, only two methods in the end produced the same result.

Assuming that points 1 and 2 were chosen at random and used in an accuracy assessment, the final upscaled results and their corresponding error matrices would be quite different. No clear rule can be set out as to which one of the two classes ("Forest" and "Other surfaces with sealed covering"), represented equally, should actually be assigned to the new pixel. Therefore, either of the two classes should be acceptable.

Point 1 would indicate that only the SM performed well in the upscaling process although, upon visual inspection, this method gives the least favourable result. On the other hand, point 2 would indicate poor upscaling for all methods, even though three out of four in fact produced good results. 


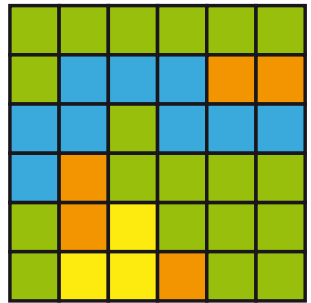

Original

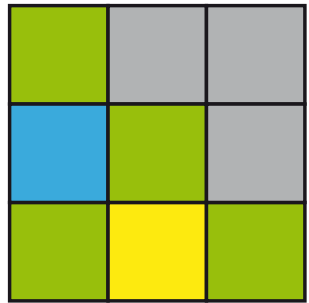

Majority

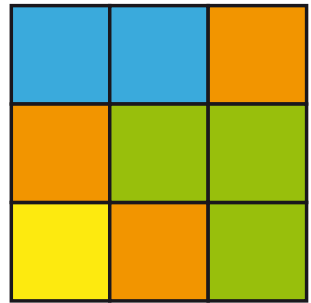

Standard NN

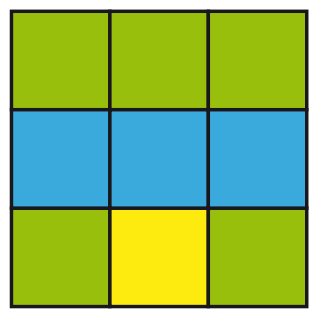

Other possible NN

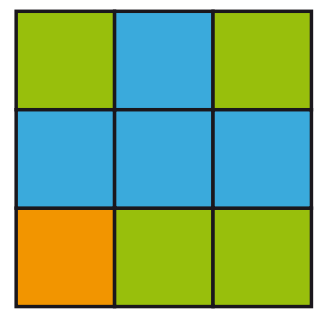

Other possible NN

Fig. (6). Example of upscaling with an even CF. Original data (upper left). Output images as a result of the "Majority method" (upper centre). The ArcMap ${ }^{\odot}$ standard outcome of the "Nearest Neighbour method" is shown in upper right and examples of other possible outcomes of the NN method at the bottom. Grey cells: ambiguous (value allocated in ArcMap ${ }^{\mathcal{O}}$ is randomly chosen from the those colors which have the same number of pixels).
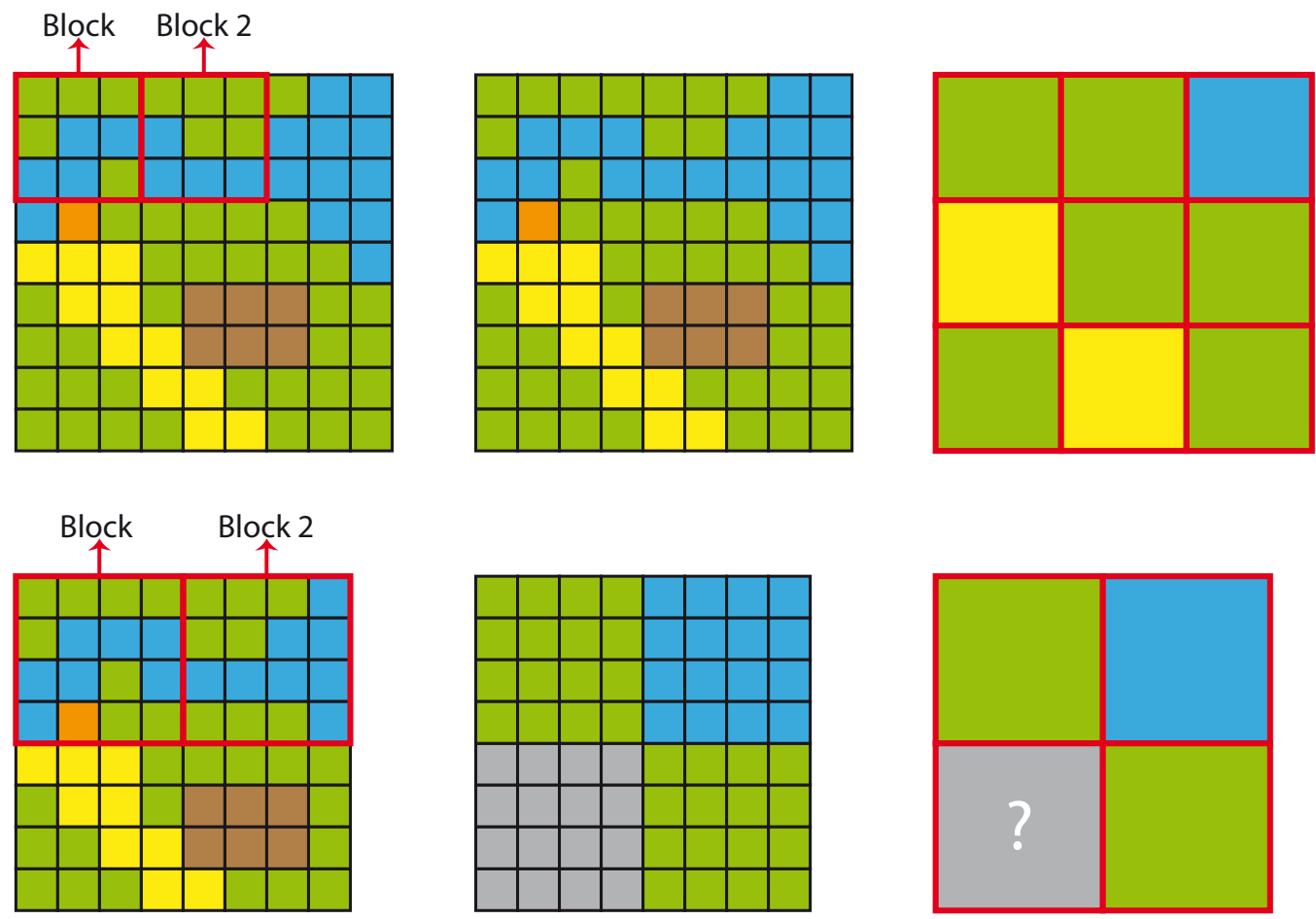

Fig. (7). Different steps of the "statistical approach". Top: 3x3 blocks. Bottom: 4x4 blocks.

It turns out, then, that point 2 is not really representative for the pixels which, after upscaling, make up one new pixel.

Although it is important to point out these shortcomings, only a few of such dubious cases actually occurred in our analysis, and it is very unlikely they had a significant influence on the accuracy assessment.

\section{RESULTS}

A visual comparison (Fig. 9) shows that the $M M$ is unable to preserve thin linear features. To a certain extent, this also applies to the SM. The MM is the one that simplifies the image the most and therefore can be useful if small features, which might add a fuzzy appearance to the 


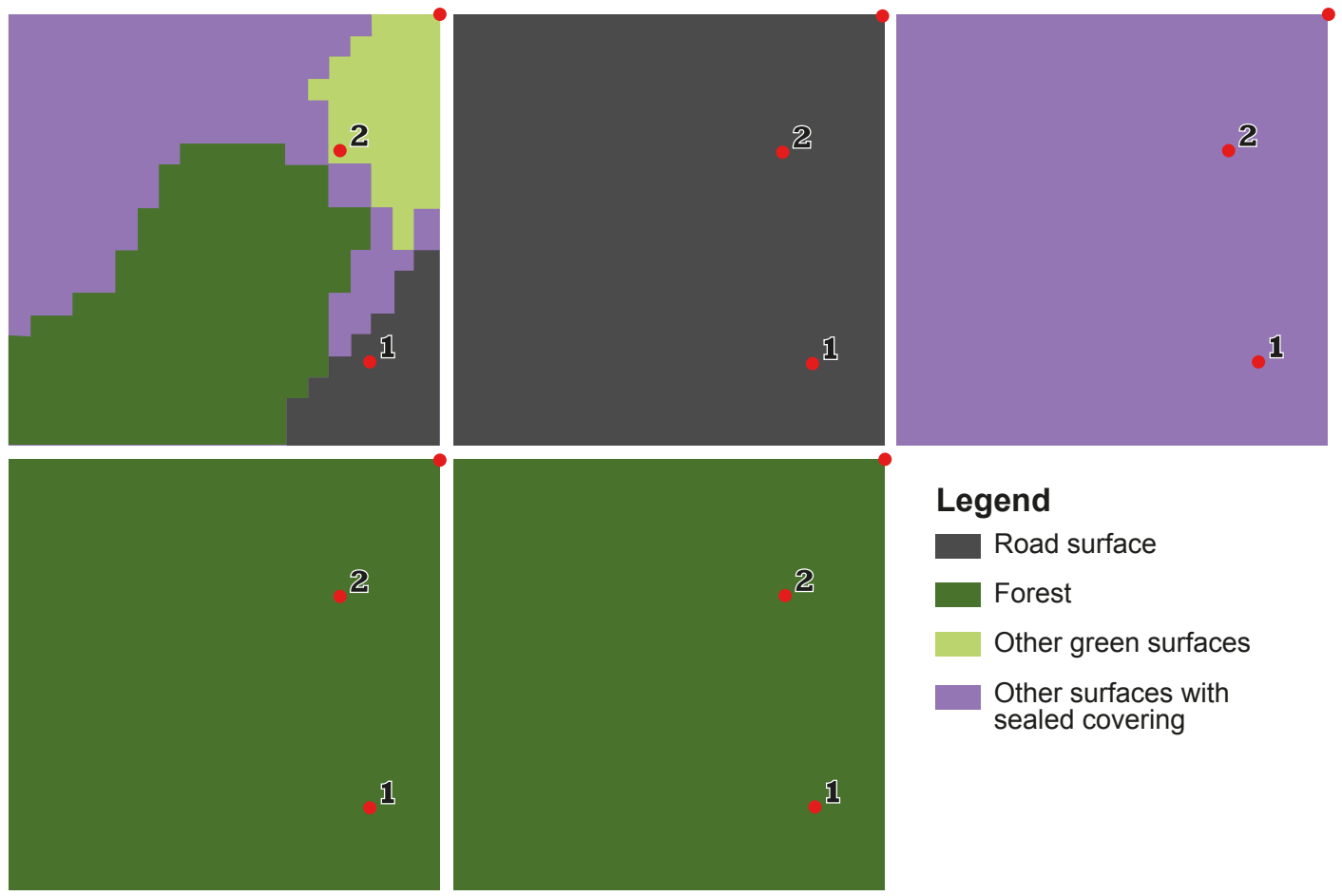

Fig. (8). Results of different upscaling methods. Numbers 1 and 2 indicate examples of randomly chosen control points. Original dataset (top left) with a frame of 20 by 20 pixels; upscaling results using the "statistical method" (top middle), the "majority method" (top right), the "nearest neighbour method" (bottom left) and the "direct method" (bottom middle).

map, should be avoided. The NM and the DM yield the best results.

The statistical evaluation gives an approximate measure of the amount of information lost due to upscaling, and is thus an indicator of the performance of the different methods. The pairwise $Z$-scores (not shown) for each of the four runs for every test area showed that most of Kappa values represented the same population with a level of significance of $95 \%$. The exception was in the Thônex region, for which $Z$-scores of two runs using the NM and four runs using the DM were not comparable. As these runs all occurred when comparing the third run to others, this run was eliminated from all subsequent calculations.

Fig. (10) summarises the common Kappa values obtained over the different runs for the different methods, test areas and thematic resolutions. Note that these values represent four runs, except for the Thônex region which is only made up of three for reasons explained above.

As expected, the spatial upscaling of the original data with its approximately 26 classes resulted in a bigger loss of information than did the same procedure on the data with the reduced classes number. This is particularly apparent in the urban areas, because a reduction in classes automatically results in a more homogenous land cover of the subset.

The connection between heterogeneity and loss of information can also be seen when comparing the different regions. The loss is least in the Satigny subset, which is dominated by agricultural parcels of large size and is thus quite homogeneous (see Fig. 2). The reason for the urban area (Genève Cité) having performed better than the also fairly urban area of Thônex might be that the subset of
Genève Cité covers also a large part of the Lake Geneva, therefore adding quite a big homogenous area (compare Fig. 2).

The common value of 1 - Kappa gives an estimate of how much information is lost during the process of changing the resolution (Fig. 10): for the upscaling based on the high thematic resolution, between $8 \%$ (Satigny), 19\% (Genève Cité) and 22\% (Thônex) of the information was lost, whereas for the agglomerated classes, it ranged from 7\% (Satigny) to $18 \%$ (Thônex). Once again, lower losses do occur in the more homogenous region of Satigny, where the difference of information loss between the two thematic resolutions is also smallest.

To evaluate the performance of the different methods, the results from each similarity assessment were compared using a $t$-test. The results for the regions Thônex and Genève Cité are shown in Table 1. The results for Satigny are not presented, as all methods in this region did not show any significant difference at all. In the table, the numbers signify how many times the results from the different runs (three runs for Thônex and four for Genève Cité) differed from one another. One can see that the "direct method" is always significantly different from the other methods for the Genève Cité region and twice for the region Thônex. Comparing this result with the bar heights in Fig. (10), one can conclude that the DM is the least favourable method, while the others do not really differ. Taking into account that the MM performed worst in the visual comparison, followed by the SM, the NN can be considered as the most satisfactory method for degrading the resolution of our land cover map from $0.25 \mathrm{~m}$ to $5 \mathrm{~m}$. 

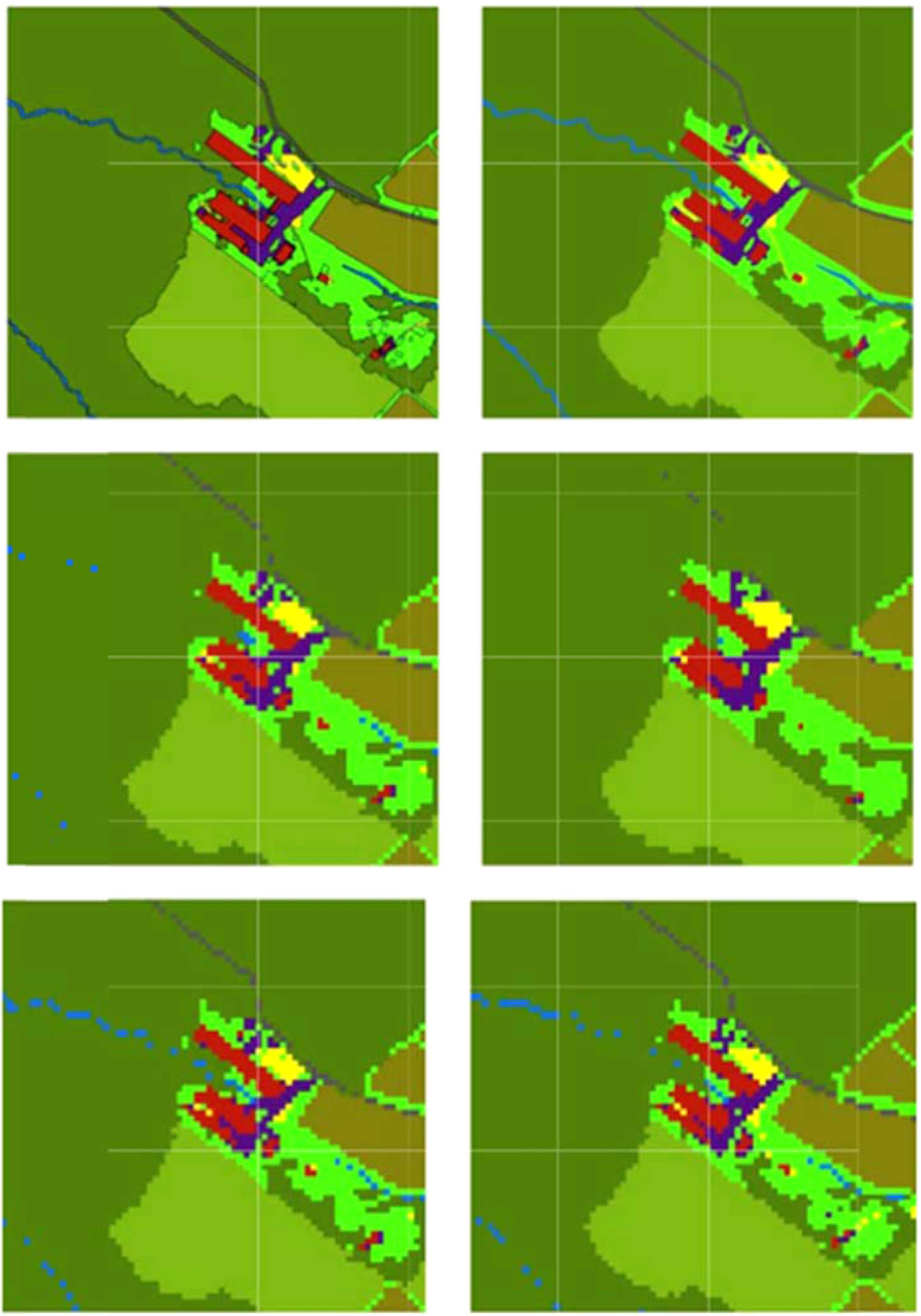

Fig. (9). Visual comparison of upscaling results: original vector data (top left), converted raster data with a resolution of $25 \mathrm{~cm}$ (top right), upscaling results using the SM (middle left), MM (middle right), NM (bottom left) and DM (bottom right).

\section{CONCLUSION}

Implemented in ArcGIS, several methods of degrading the resolution of a land cover map from $0.25 \mathrm{~m}$ to $5 \mathrm{~m}$ (cell factor $=20$ ) were evaluated under the criterion of minimizing the information loss and preserving thin linear features as much as possible. Except for the "direct method", which is the least favourable one, all other methods performed almost equally well according to the statistical analysis. From the visual interpretation, it can be seen that thin linear features, such as small roads and rivers are lost when using the "majority method". Summing up the results from all the analyses, the "nearest neighbour" method performed best, closely followed by the "majority" and "statistical" methods.

For a given method, information loss, expressed by 1 Kappa, is strongly related to the heterogeneity of the land cover, so that it is the least in the rural Satigny region amounting to approximately $8 \%$ (original classes) and $7 \%$ (agglomerated classes). Information loss was the highest in the mixed urban / suburban Thônex area (22\% original classes and 18\% agglomerated classes). Values for urban Genève Cité lie in between, owing to the presence of the lake area. 


\section{Common Kappa Value}

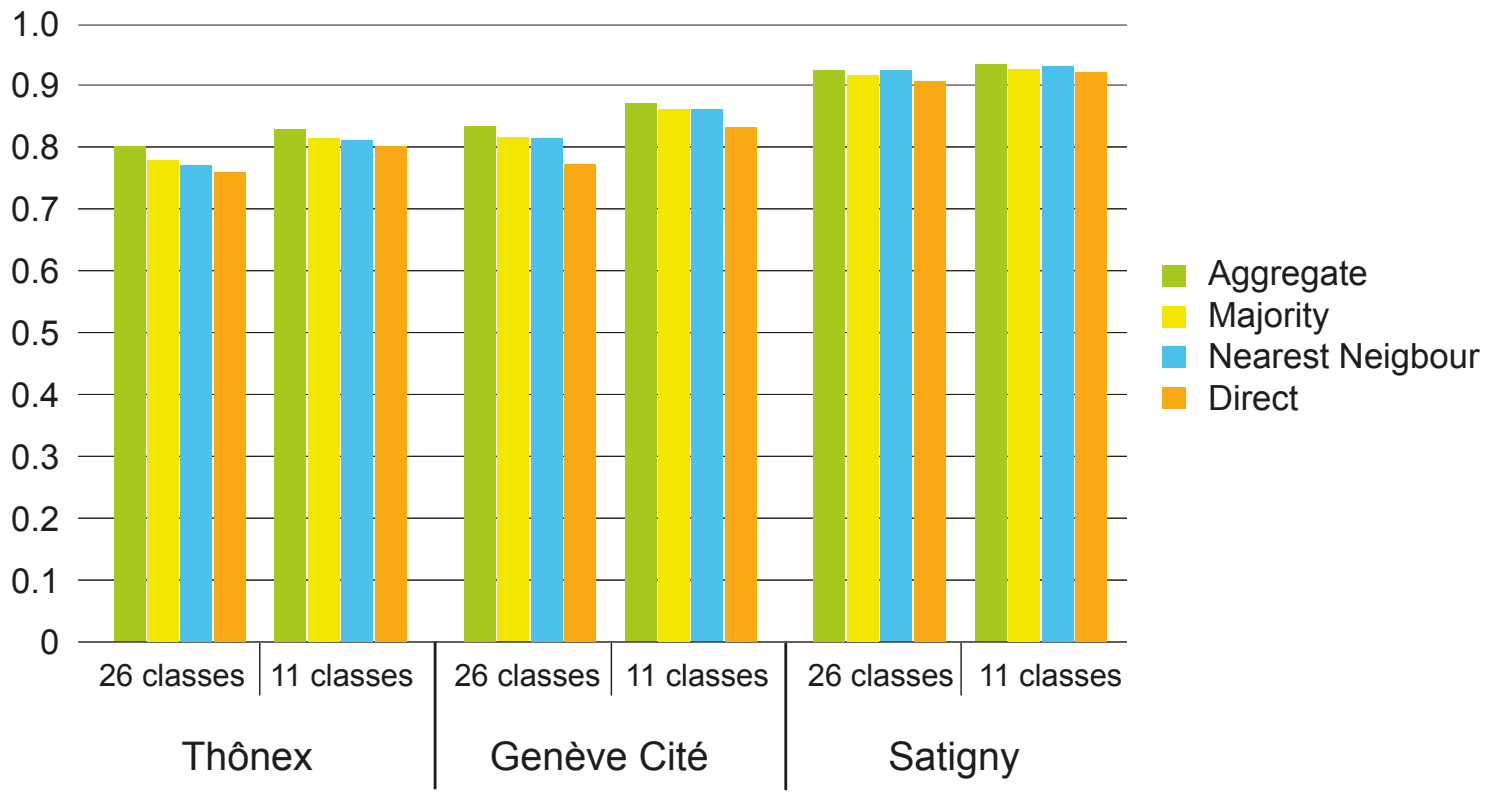

Fig. (10). Common Kappa value for different methods, test areas and thematic resolutions.

Table 1. Comparison of different upscaling methods for Thônex and Genève-Cité subsets, and for intial and reduced numbers of classes. Numbers signify how many times the results from the different runs (three runs for Thônex and four for Genève Cité) differed from one another.

\section{Thônex}

\begin{tabular}{lccccc}
\hline \multicolumn{1}{c}{ Thematic classes: } & \multicolumn{1}{c}{ Initial } & \multicolumn{2}{c}{ Reduced } \\
\hline \multicolumn{1}{c}{ Method: } & DM & NN & MM & DM & NN \\
\hline Nearest Neighbour & $\mathbf{0}$ & & & $\mathbf{0}$ & $\mathbf{0}$ \\
\hline Majority & $\mathbf{0}$ & $\mathbf{0}$ & $\mathbf{0}$ & $\mathbf{1}$ & $\mathbf{0}$ \\
\hline Statistical & $\mathbf{1}$ & $\mathbf{1}$ & $\mathbf{0}$ & $\mathbf{0}$ \\
\hline
\end{tabular}

\begin{tabular}{|c|c|c|c|c|c|c|}
\hline \multirow{3}{*}{$\begin{array}{c}\text { Thematic classes: } \\
\text { Method: }\end{array}$} & \multicolumn{6}{|c|}{ Genève Cité } \\
\hline & \multicolumn{3}{|c|}{ Initial } & \multicolumn{3}{|c|}{ Reduced } \\
\hline & $\mathrm{DM}$ & $\mathrm{NN}$ & MM & DM & $\mathrm{NN}$ & MM \\
\hline Nearest Neighbour & 3 & & & 1 & & \\
\hline Majority & 2 & $\mathbf{0}$ & & 1 & $\mathbf{0}$ & \\
\hline Statistical & 4 & $\mathbf{0}$ & 1 & 3 & $\mathbf{0}$ & $\mathbf{0}$ \\
\hline
\end{tabular}

The results obtained in this study are, to a certain extent, site- and criterion-specific. Under different conditions of land cover texture, or without the need of preserving linear features, the ranking of methods could be different. Therefore, a testing procedure such as proposed here, combining visual inspection and statistical analysis, should be conducted prior to any attempt at spatial degradation of a land cover map.

\section{CONFLICT OF INTEREST}

The author confirm that this article content has no conflicts of interest.

\section{ACKNOWLEDGEMENT}

Declared none.

\section{REFERENCES}

[1] Moukana JA, Koike K. Geostatistical model for correlating declining groundwater levels with changes in land cover detected from analyses of satellite images. Comput Geosci 2008; 34:1527-40.

[2] Valentin C, Agus F, Alamban R, et al. Runoff and sediment losses from 27 upland catchments in Southeast Asia: impact of rapid land use changes and conservation practices. Agric Ecosyst Environ 2008; 128: 225-38

[3] Cebecauer T, Hofierka J. The consequences of land-cover changes on soil erosion distribution in Slovakia. Geomorphology 2008; 98: 187-98.

[4] Chapin FS, Zavaleta ES, Eviner VT, et al. Consequences of changing biodiversity. Nature 2000; 405: 234-42.

[5] Pongratz J, Bounoua L, DeFries RS, et al. The impact of land cover change on surface energy and water balance in matogrosso, Brazil Earth Interact 2006; 10:1-17.

6] Rotenberg E, Yakir D. Distinct patterns of changes in surface energy budget associated with forestation in the semiarid region. Glob Change Biol 2001; 17(4) : 1536-48. 
[7] Lambin EF, Strahler AH. Change-Vector analysis in multitemporal space: a tool to detect and categorize land-cover change processes using high temporal-resolution satellite data. Remote Sens Environ 1994; 48: 231-44.

[8] Yuan F, Sawaya KE, Loeffelholz BC, Bauer ME. Land cover classification and change analysis of the Twin Cities (Minnesota) Metropolitan Area by multitemporal Landsat remote sensing. Remote Sens Environ 2005; 98: 317-28.

[9] Mas J-F. Monitoring land-cover changes: a comparison of changedetection techniques. Int J Remote Sens 1999; 20(1): 139-52.

[10] European Environment Agency. Land cover flows based on Corine land cover changes database (1990-2000). 2011.

[12] Hay GJ, Niemann KO, Goodenough DG. Spatial Thresholds, Image-Objects and Upscaling: a Multi-Scale Evaluation. Remote Sens Environ 1997; 62: 1-19.

[13] Gupta RK, Prasad TS, Krishna RPV, Bala Manikave PM. Problems in upscaling of high resolution remote sensing data to coarse spatial resolution over land surface. Adv Space Res 2000; 26(7): 1111-21.

[14] AGGLO. Projet d'agglomération franco-valdo-genevois. http://www.projet-agglo.org/ http://www.eea.europa.eu/data-and-maps/data/land-cover-flows-based-on-corine-land-cover-changesdatabase-1990-2000-1. 2011
[15] Schwarzer S, Allenbach K, Jaquet J-M. Cartographie semi-automatisée et multi-sources de la couverture du sol. 1. Périmètre de l'Agglomération franco-valdo-genevoise (CCSA04, moyenne résolution). Arch Sci Gen 2009; 62: 107-24.

[16] SITG. Description of multisource landcover mapping methodology. Available at http://etat.geneve.ch/geoportail/metadataws/Publish/documents/CS_ANALYSE_SPECTRALE_SOL.pdf 2011

[17] Harayama A, Jaquet J-M. Cartographie semi-automatisée et multisources de la couverture du sol dans le Canton de Genève. Géomatique Suisse 2004; 8: 503-6.

[18] Niggeler L, Jaquet J-M. Cartographie à haute résolution de la couverture du sol à partir d'images aériennes. INFO D+M 2007; 2 : 15-16.http://www.cadastre.ch/internet/cadastre/fr/home/docu/info vd.html

[19] SITG.Couverture du sol par analyse spectrale (basse). Available at http://etat.geneve.ch/sitg/dictionnaire.html 2011

[20] Jenness J, Wynne JJ.Kappa analysis (kappa_stats.avx) extension for ArcView 3.x. Jenness Enterprises. Available at http://www.jennessent.com/arcview/kappa_stats.htm 2011

[21] Foody GM. Status of land cover classification accuracy assessment. Remote Sens Environ 2002; 80: 185-201.

(C) Hubert et al.; Licensee Bentham Open.

This is an open access article licensed under the terms of the Creative Commons Attribution Non-Commercial License (http://creativecommons.org/licenses/by-nc/3.0/) which permits unrestricted, non-commercial use, distribution and reproduction in any medium, provided the work is properly cited. 